\title{
RANKING Y ESTADO DE LA INVESTIGACIÓN EN LAS FACULTADES DE DERECHO EN COLOMBIA*
}

\section{Ranking and situation of the investigation in the faculties of law in Colombia}

\author{
Antonio Milla** \\ Recepción: 24 de abril de 2018. Aceptación: 10 de junio de 2018. \\ DOI: http://dx.doi.org/10.21017/Rev.Repub.2018.v25.a51
}

\section{RESUMEN}

Este artículo discute las características y el estado de la investigación jurídica y sociojurídica que se realiza en el marco de los programas de Derecho en Colombia. Se centra en cuestiones clave relativas a la calidad de los programas de educación jurídica existentes en el país. Para este propósito, con base en los resultados de la convocatoria nacional Colciencias 2017 para reconocer y categorizar grupos de investigación en Colombia, se emprende una clasificación escalonada o ranking de los programas de Derecho. El resultado de este ranking ofrece información sobre el papel de las universidades públicas y privadas en la investigación, la distribución regional de la producción de investigación, las correlaciones entre investigación y programas de doctorado, las relaciones entre la investigación y la acreditación de alta calidad de los programas de Derecho, así como las conexiones entre la investigación y la obtención del registro calificado de programas académicos en esta disciplina. Las conclusiones se centran en aspectos relacionados con las políticas de educación superior y sus efectos sobre las prácticas y el estado de la investigación jurídica en Colombia.

Palabras clave: sociología del derecho; educación; educación jurídica; investigación jurídica; investigación sociojurídica; calidad de la educación.

* Artículo producto de trabajo investigativo adscrito al Grupo de Investigación en Historia, Conflictos y Cambio Social del Instituto Latinoamericano de Altos Estudios (ilae). Bogotá, D. C.

** Administrador de Empresas del Instituto Peruano de Administración de Empresas -IPAE-, profesor universitario, editor académico, director e investigador del Instituto Latinoamericano de Altos Estudios -ILAE-. Este trabajo está adscrito al Grupo de Investigación en Historia, Conflictos y Cambio Social del ILAE. 


\section{SUMMARY}

This article discusses characteristics and the state of legal and socio-legal research undertaken in the framework of law degree programs in Colombia, focusing on key issues concerning the quality of the country's present legal education programs. For this purpose, based on the results of the 2017 Colciencias nationwide call to recognize and categorize research groups in Colombia, a ranking of law degree programs is undertaken. The output of this ranking offers insights about the role of private and public universities in research, the regional distribution of research production, the correlations between research and $\mathrm{PhD}$ programs, the relationships between research and high-quality accreditation of law degree programs, as well as the connections between research and obtaining the qualified register of academic programs. Conclusions focus on aspects concerning higher education policies and their effects on practices and the status of legal research in Colombia.

Key words: sociology of law; education; legal education; legal research; sociolegal research; quality in education.

\section{INTRODUCCIÓN}

Este trabajo contiene un escalafón o ranking de la investigación científica desarrollada en las facultades de Derecho en Colombia, elaborado con base en los datos provenientes de los resultados definitivos de la convocatoria del Departamento Administrativo de Ciencia, Tecnología e Innovación (Colciencias), correspondientes a 2017, que han sido publicados en su página web $^{1}$. La construcción del escalafón es un medio para elaborar un mapa de la investigación jurídica y sociojurídica nacional, lo que permite emprender algunos análisis sobre el estado y las características de la investigación y, a su vez, relacionarlos con instrumentos empleados para evaluar la calidad de la educación superior ${ }^{2}$.

Se supone, probablemente con tino, que la investigación docente es uno de los principales indicadores de la calidad de la educación, como quiera que ella permite renovar los contenidos de los programas académicos y orientarlos a la

$1 \mathrm{Al}$ respecto, se puede ver Colciencias. Listado resultados finales -781 de 2017- Grupos (Para Consulta), en www.colciencias.gov.co, revisado en enero de 2018.

2 Una visión de las relaciones entre la investigación y la enseñanza del Derecho en Henry Bocanegra. «La enseñanza del Derecho y la formación de los abogados», en Revista Republicana, nro. 12, 2012, pp. 327 y ss. 
solución de problemas de la sociedad y del conocimiento 3 . Por ello, la investigación es una de las condiciones de calidad que debe ser verificada para el otorgamiento del registro calificado a los programas académicos, es decir, de la autorización del Estado para que puedan ser ofrecidos y desarrollados. Así mismo, es un factor de calidad para ponderar la acreditación de los programas académicos y de las instituciones de educación superior, esto es, del reconocimiento que hace el Estado en cuanto a que se trata de programas o de instituciones de alta calidad, caracterizados por la excelencia.

Los parámetros usados por Colciencias para clasificar los grupos de investigación han sido objeto de discusión y debate $\mathrm{y}$, sin duda, varios de ellos son materia de grandes reservas ${ }^{4}$. Sin embargo, este no es el escenario para adelantar semejante discusión, puesto que los fines del trabajo son muy diferentes. Con todo, cabe señalar que buena parte de las críticas tienen que ver con la utilización de varios criterios que pueden ser aplicados a la evaluación de la investigación en ciencias básicas, ingenierías y ciencias de la salud, pero que resultan excesivos e inapropiados respecto de las ciencias sociales, aún más, cuando se trata de la investigación jurídica; pero pese a lo anterior, puede decirse a favor de los propósitos perseguidos por este trabajo que, en últimas, aquí los grupos de investigación jurídica y sociojurídica no se comparan con los de otras disciplinas y que todos los grupos fueron sopesados con el mismo rasero. Lo primero significa que no se puede derivar ninguna inequidad, por el uso de parámetros más favorables a otras disciplinas, pues no hay ninguna comparación con ellas. En cuanto a lo segundo, si se acepta que los criterios de Colciencias son desproporcionados y excesivos, el lector solo deberá considerar que alcanzar resultados muy positivos es aún más meritorio, pero esto no invalida el análisis de los resultados de la aplicación de los parámetros de Colciencias.

De otra parte, es preciso tener en cuenta que, de acuerdo con sus criterios, los cuales pueden ser consultados en la página web de Colciencias, la calidad de los grupos de investigación es definida por un escalafón en el cual la categoría

3 Existen pocos estudios sobre el impacto de la investigación docente en la enseñanza del Derecho. Uno de ellos en Nora Alba Cossio Acevedo. «Informe final de la investigación: Pertinencia e impacto social de la investigación jurídica: el caso del programa de dere- cho de la Universidad de Antioquia 1992-2009», en Estudios de Derecho, vol. 70, nro. 156, 2013, pp. 163-182. Desde luego, los resultados de la repercusión varían de programa a programa, según la cultura investigativa de profesores y estudiantes, la frecuencia del uso transversal de la producción investigativa en el aula y la calidad de los productos de investigación de los docentes.

4 El documento que expone los parámetros de Colciencias se puede consultar en www.colciencias.gov.co, 
A1 es la más encumbrada y sobresaliente. A continuación concurre la categoría A, que vendría a ser la segunda más alta y, junto a la anterior, refleja una situación de excelencia. Enseguida, se encuentra la categoría B, estimada como bastante destacada, pues, tanto Colciencias como el Consejo Nacional de Acreditación -cna-, conforme a criterios que se expondrán más adelante, la consideran de alta calidad. Luego aparece la categoría $\mathrm{C}$, positiva, pero no muy prominenteo descollante. Son, en consecuencia, cuatrolosescalafones existentes. Después de los mencionados, un grupo puede ser apenas reconocido, lo que traduce que es en efecto un grupo de investigación, pero nada relevante. A su vez, un grupo puede no ser siquiera reconocido, lo que significaría que hay un grupo con pretensiones en la materia, pero todavía no de investigación, pues no tiene resultados. A los dos últimos no se hará referencia.

El escalafón de la investigación en las facultades de Derecho en Colombia se construyó con fundamento en las categorías asignadas a los grupos por Colciencias. Para hacer el ranking se tuvo en cuenta el número de grupos en categoría A1. Entre las instituciones con grupos en categoría A1, solo se consideró la presencia de grupos en A, B o C para resolver desempates. Con posterioridad, en los mismos términos, fueron consideradas en su orden las demás categorías de grupos, al usar las subsiguientes para dilucidar empates.

Es importante revelar que solo fueron revisados los datos definitivos de la Convocatoria 781 de 2017 de Colciencias para reconocer y categorizar grupos de investigación, no los datos de anteriores convocatorias como, por ejemplo, la Convocatoria de 20155 . La elaboración del ranking y el análisis de los datos exigían una información con un único referente, de tal manera que pudieran hacerse comparaciones enigualdad de condiciones. Desdeluego, los resultados de 2017 son los más actualizados. Todas las instituciones de educación superior y grupos tuvieron la oportunidad de presentarse a la convocatoria de 2017.

Hay algunos grupos de investigación que tienen por objeto principal de investigación al Derecho, pero no hacen parte de instituciones de educación superior que ofrezcan y desarrollen programas de Derecho, por lo que no fueron tenidos en cuenta, pues este trabajo se refiere a la investigación en las facultades y programas de Derecho colombianos. En el caso de instituciones con algún grupo localizado en una ciudad distinta a aquella donde se ofrecen los estudios de Derecho, este no se tuvo en cuenta, dado que las posibilidades de impactar a distancia a un programa académico de otra ciudad son

5 Los grupos que no se presentaron a la convocatoria del 2017 pierden en mayo del 2018 la categoría que obtuvieron en la convocatoria del 2015, razón por la cual no fueron considerados. 
muy limitadas. Cuando se presentaba la situación de un grupo de investiga- ción a cargo de dos instituciones diferentes, solo contaba a favor de la insti- tución en la que coincidía la sede del grupo y del programa, con un valor de 0.5. Surgió un programa con dos grupos de investigación en categoría C respecto de los cuales no fue indicado en Colciencias la ciudad sede, lo que hacía imposible identificar el programa al cual apoyaban, razón por la cual no fueron tenidos en cuenta.

Para efectos de identificar a los grupos como pertenecientes al área del Derecho, fue considerada la definición que, con ese propósito, hace cada institución de educación superior al registrar sus grupos, y el programa al cual están adscritos. De este modo, salvo información más precisa, algunos grupos que representan una auténtica miscelánea de las áreas del conocimiento más diversas, que podían incluir al Derecho en su nombre junto a otras muchas materias, pero que al indicar el área no señalaban al Derecho, no fueron tenidos en cuenta.

El propósito del ranking, que en sí mismo solo tiene un valor puramente descriptivo, es utilizarlo como un medio para analizar el estado de la investigación y la calidad de la educación jurídica en Colombia y, a partir de allí, sacar algunas conclusiones sobre la realidad nacional ${ }^{6}$. Luego, a fin de cuentas, lo queabarca el trabajoesunainspección sobrela calidad dela educaciónjurídica7 . La calidad tiene un impacto sobre las posibilidades de éxito profesional; en consecuencia, de movilidad social, es decir, tiene implicaciones sociales y políticas importantes ${ }^{8}$.

Este es un trabajo de sociología jurídica, que también podría ser ubicado en el campo de la educación, elaborado con fuentes documentales, con aplicaciones

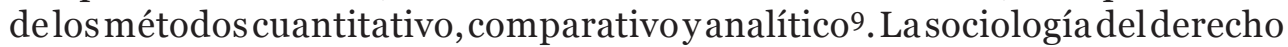

6 Un marco teórico sobre la educación jurídica y la investigación, en Ramón Larrauri Torreola. «La educación jurídica como campo de investigación desde una conceptualización epistemológica », Universitas. Revista de Filosofía, Derecho y Política, nro. 3, 2005/ 2006, pp. 61-96.

7 Sobre la importancia de la investigación, Norhys Esther Torregrosa Jiménez y Rodolfo Torregrosa Jiménez. «Editorial. La investigación sociojurídica una función prioritaria en la formación de los abogados y abogadas del siglo XXI en Colombia», Verba Iuris, nro. 28, 2012, pp. 13-15.

8 El problema es explorado en María Adelaida Ceballos Bedoya. «Educación jurídica, abogados y movilidad social en Colombia», Universidad Nacional de Colombia, Facul- tad de Humanidades, Departamento de Sociología, trabajo de investigación Maestría en Sociología, 2016, pp. 49 y ss.

9 Las cuatro posiciones en el ranking A1, A, B y C corresponden a modelos ideales o tipificaciones de la organización y desarrollo de la investigación, donde a cada posición corresponden unos determinados atributos. Enseguida, es posible hacer comparaciones 
se ha ocupado, como uno de sus temas prioritarios, de la educación jurídica, y a ella se dedica este texto al intentar evaluarla a través de la investigación producida en las facultades de Derecho. La importancia de la educación jurídica, para convertirla en objeto de estudio de la sociología del derecho, parte del supuesto según el cual de acuerdo con las características de la educación de los operadores del Derecho habrá determinadas clases de abogados lo que, a su vez, revertirá sobre la práctica del Derecho, tema último al que por excelencia se dedica la sociología jurídica ${ }^{10}$. Por su parte, para la educación como disciplina del conocimiento, la trascendencia de la investigación se desprende de la importancia que alcanza para inducir condiciones de calidad en los procesos formativos ${ }^{11}$.

\section{DESCRIPCIÓN DE LOS DATOS Y OBSERVACIONES}

Se observa que solo nueve facultades de Derecho poseen grupos en la categoría A1, la más alta que existe en el escalafón ideado por Colciencias. Las únicas con dos grupos en ese nivel son la Universidad Colegio Mayor del Rosario y la Universidad de Los Andes. Las demás facultades registran solo un grupo en ese escalafón por institución. Existen, entonces, 11 grupos de investigación que muestran resultados de excelencia avanzados. Estos serían los grupos de investigación, conforme a los parámetros de Colciencias, más importantes de la comunidad jurídica nacional. Los programas de Derecho que registran grupos A1 representan 4,8\% del total de programas de la disciplina, considerando que a noviembre de 2017 había 184 en el país ${ }^{12}$.

En orden alfabético, los 11 grupos pertenecen a la Universidad de Caldas, la Universidad Católica de Colombia, la Universidad Colegio Mayor de Nuestra Señora del Rosario, la Universidad Javeriana (Bogotá), el icesi, la Universidad de Los Andes, la Universidad Nacional de Colombia, la Universidad del Norte

entre cada una de las posiciones. De este modo, se da aplicación al método comparativo expuesto por Max Weber. Economía y sociedad, 2. ${ }^{\mathrm{a}}$ ed., México D. F., Fondo de Cultura Económica, 1992, pp. 16 a 18.

10 Germán Silva García. El mundo real de los abogados y de la justicia, t. iv, «Las ideolo- gías profesionales», en Bogotá, Instituto Latinoamericano de Servicios Legales Alterna- tivos ilsa- y Externado, 2001, pp. 26 y ss.

11 Acerca de algunos marcos teóricos y referentes del desarrollo de la investigación jurídi- ca, que no es una línea que haya sido explorada en este trabajo, puede consultarse a Andrés Botero Bernal. «Un marco de comprensión del avance de la investigación jurídi- ca en Colombia», Academia. Revista sobre la Enseñanza del Derecho, año 5, nro. 10, 2007, pp. 35-70.

12 Al respecto véase Ministerio de Educación Nacional. Sistema Nacional de Información de la Educación Superior -snies-, consultando en www.mineducacion.gov.co. 
y la Universidad Sergio Arboleda de Santa Marta. Son nueve universidades y ninguna institución universitaria ${ }^{13}$. De las nueve instituciones, siete son privadas y solo dos son públicas. Esto último coincide, para consolidar una tendencia, con otros datos que se considerarán más adelante. Esa tendencia indica un rezago importante de la universidad pública. En cuanto a la ubicación de los nueve programas dueños de esos grupos de investigación, como era de esperarse, predomina Bogotá con cinco, mientras Cali, Barranquilla, Manizales y Santa Marta poseían uno en cada caso. Con todo, como se trata de una élite de grupos de investigación, por ende, un grupo muy pequeño, sería prematuro hacer cábalas acerca de la distribución regional de los procesos de producción del conocimiento. Esto podrá inspeccionarse mejor al considerar la ubicación regional del total de grupos en categoría A1 y A.

Es evidente que entre los grupos de calidad avanzada predomina, de manera absoluta, el derecho público como área o campo de trabajo ${ }^{14}$. Esto, además, coincide con las tendencias de la educación jurídica contemporáneas y con las mayores demandas del mercado de trabajo. Ciertamente, la expansión del Estado intervencionista, el impulso logrado por la figura del Estado social de derecho, el importante rol que juega el Estado como el mayor empleador, la relevancia delas acciones constitucionales y la constitucionalización del Derecho en todas sus variantes, han incidido de modo directo para que los derechos constitucional y administrativo ocupen un lugar prioritario ${ }^{15}$. Lo descrito, por ende, denota que existe una congruencia total entre las tendencias de la educación jurídica a nivel nacional e internacional, quele conceden una importancia prioritaria al derecho constitucional y al derecho administrativo, y la investigación de excelencia en los programas de Derecho colombianos. A la par, también existe esa misma concatenación entre las exigencias del mundo de la profesión de abogado, que por igual requiere de un mayor número de profesionales y de más conocimientos vinculados a esos mismos campos del derecho público, y la investigación de excelencia en los programas de Derecho nacionales.

13 El sistema educativo colombiano tiene una absurda y excluyente división entre universidades e instituciones universitarias, lo que en términos prácticos acarrea -tan solo- que las segundas no puedan ofrecer programas de maestría ni de doctorado. Dado que la dirección de trabajos de grado en maestría y doctorado cuenta entre los parámetros que dispensan puntaje, sin duda, es muy difícil que una institución universitaria pueda poseer un grupo en categoría «A1». Sobre la prescripción legal que introduce la distin- ción véase Instituto Colombiano para el Fomento de la Educación Superior -icfes-. Educación superior. Compendio de normas, Bogotá, icfes, 1995, pp. 11 a 13.

14 La conclusión anterior deriva de la revisión de las líneas de investigación y producción de los grupos A1.

15 Germán Silva García. «Prospectivas sobre la educación jurídica», en Rogelio Pérez Perdomo y Julia Cristina Rodríguez (coords.). La formación jurídica en América Latina, Bogotá, Externado, 2006. 
Para efectos del ranking de la investigación en Derecho que se ha elaborado, la Universidad Colegio Mayor de Nuestra Señora del Rosario ocupa la posición de punta con cinco grupos en A1 y A. La plaza subsiguiente es encabezada por el programa de la Universidad de Los Andes, merced a la tenencia de dos grupos en A1, junto a otro en A. La siguiente posición la ocupa la Facultad de Derecho de la Universidad Católica de Colombia, pues tiene un grupo en categoría A1, al lado de otros tres en A. Por tanto, el ranking identifica así las primeras posiciones: Universidad Colegio Mayor de Nuestra Señora del Rosario (1. $\left.{ }^{\circ}\right)$, Universidad de Los Andes $\left(2 .^{\circ}\right)$, Universidad Católica de Colombia $\left(3 .^{\circ}\right)$.

De acuerdo con lo anterior, los tres programas de Derecho con mayor desarrollo de la investigación, de conformidad con los parámetros de Colciencias, que alcanzarían cotas de excelencia, corresponden a tres universidades privadas. Adicional a lo anterior, los tres programas se desarrollan en la capital del país, esto es, en Bogotá. Todas son universidades laicas.

Comparecen con un grupo A1 y dos A las universidades Nacional de Colombia $\left(4 .^{\circ}\right)$, la cual también tiene un grupo reconocido, y Javeriana de Bogotá $\left(5 .^{\circ}\right)$, donde la primera resulta con ventaja por la tenencia de un grupo en el nivel B. Luego, con un grupo en A1, concurren la Universidad del Norte (6. ${ }^{\circ}$ ), la Universidad de Caldas $\left(6 .^{\circ}\right)$, que tiene además un grupo reconocido, y la Universidad icesi $\left(6 .^{\circ}\right)$. Después comparece la Universidad Sergio Arboleda de Santa Marta $\left(9 .{ }^{\circ}\right)$, con un grupo A1, compartido con otra institución. Para cerrar, aparece la Universidad Externado de Colombia, que no cuenta con ningún grupo en A1, pero de modo meritorio tiene una cantidad bastante elevada de grupos en $\mathrm{A}$ y $\mathrm{B}^{16}$.

Las tres instituciones empatadas en el sexto lugar, al tener un único grupo de investigación en A1, y la Sergio Arboleda de Santa Marta en el noveno puesto, con medio grupo en A1, sobrepasaron a varias universidades que presentaban simultáneamentevarios grupos en categorías A y B, pero que notenían ninguno en la categoría superior. Pese a lo cual, es probable que, en un plano cualitativo, las que fueron aventajadas por el motivo explicado tengan niveles de desarrollo de la investigación más sólidos, lo que será objeto de análisis más adelante.

El conjunto anterior, retratado en la tabla I, incluye las únicas nueve instituciones con grupos de investigación en A1, categoría que, por ello mismo, ni siquiera va a volver a registrarse en las tablas posteriores previstas para el reporte de datos.

16 Tiene un grupo en «C» compartido, el cual es registrado con un valor de o,5. 


\section{Ranking de la investigación en los programas de Derecho}

Tabla I. Posiciones 1 a 10

\begin{tabular}{clcccc}
\hline Ubicación & Institución & A1 & A & B & C \\
\hline $1^{\circ}$ & Colegio del Rosario & 2 & 3 & 0 & 0 \\
$2^{\circ}$ & Los Andes & 2 & $\mathbf{1}$ & 0 & 4 \\
$3^{\circ}$ & Católica de Colombia & $\mathbf{1}$ & 3 & $\mathbf{1}$ & $\mathbf{1}$ \\
$4^{\circ}$ & Nacional de Colombia & $\mathbf{1}$ & 2 & $\mathbf{1}$ & 3 \\
$5^{\circ}$ & Javeriana de Bogotá & $\mathbf{1}$ & 2 & 0 & $\mathbf{1}$ \\
$6^{\circ}$ & Norte & $\mathbf{1}$ & 0 & 0 & 0 \\
$6^{\circ}$ & Caldas & $\mathbf{1}$ & 0 & 0 & 0 \\
$6^{\circ}$ & ICESI & $\mathbf{1}$ & 0 & 0 & 0 \\
$9^{\circ}$ & Sergio Arboleda,Santa Marta & 0,5 & 0 & 0 & 0 \\
$10^{\circ}$ & Externado de Colombia & 0 & 5 & 4 & 5,5 \\
\hline
\end{tabular}

Fuente: Elaboración propia.

Los diez primeros programas de Derecho en investigación corresponden a ocho instituciones privadas y dos públicas. Esto significa que el país necesita reproducir y multiplicar el modelo de la Universidad Nacional de Colombia que se aplica en el programa de Derecho, el cual muestra enormes fortalezas y destaca entre los mejores del país, lo cual demanda un aporte sustantivo de recursos por parte del Estado. Seis de ellos están radicados en Bogotá.

En lo que concierne a la presencia de grupos de investigación en categoría A1 y A, esto es, de grupos de excelencia, se encuentran varios programas que reúnen entre uno y cinco grupos de investigación de ese alto nivel. Estos grupos, algunos de los cuales, como ya se explicó, son superiores con una categoría A1, representan de acuerdo con los parámetros de Colciencias a lo más granado y selecto de la investigación jurídica y sociojurídica del país (Curvelo, Jose,2018) .

Un grupo de investigación alcanza la categoría A cuando reúne un conjunto dispendioso de requisitos, el primero de los cuales es relativo, pues depende del puntaje alcanzado por otros grupos a nivel nacional ${ }^{17}$.

17 Tales requisitos son: 1 . Tener un indicador de grupo que le permita estar en o por encima del cuartil 2 (50\% superior); 2 . Tener un indicador de productos Top o «A» mayor que cero; 3. Poseer un indicador de productos de apropiación social del conocimiento mayor que cero; 4. Disponer de un indicador de productos o de actividades relacionadas con formación de recurso humano tipo «A», mayor que cero; 5. Contar con un investiga- dor sénior o asociado; 6. Sumar un indicador de cohesión mayor que cero; 7. Contabili- zar más de cinco años de existencia. Colciencias. «Modelo de medición de grupos de 
Programas de Derecho de las universidades de Caldas, Cartagena, Católica de Colombia, Colegio Mayor de Nuestra Señora del Rosario, de la Costa, Externado de Colombia, icesi, Javeriana (Bogotá), Libre (Barranquilla), Libre (Bogotá), Li- bre (Cali), La Sabana, Los Andes, Manizales, Manuela Beltrán, Medellín, Nacio- nal de Colombia, Norte, San Buenaventura (Cali), Santo Tomás (Bogotá), Santo Tomás (Tunja), Sergio Arboleda (Bogotá), Sergio Arboleda (Santa Marta), Simón Bolívar de Barranquilla, Simón Bolívar (Cúcuta), Sinú (Montería) y Surcolombiana tienen grupos de investigación en categorías A1 y/o A. En Derecho, ninguna institución universitaria tiene un grupo de investigación en las máximas categorías. En total son 27 los programas de Derecho que cuentan con grupos en categoría A1 y A. En noviembre de 2017 había 184 programas de Derecho activos en Colombia. De este modo, solo el 15\% de dichos programas nacionales, en distintos grados, había alcanzado la excelencia académica en el ámbito investigativo. Los anteriores, desde luego, son los 27 programas de Derecho que ejercen el liderazgo de la investigación a nivel nacional.

Una tasa como la expuesta, sin duda, es considerablemente baja. Sin embargo, para un diagnóstico que relacione el desarrollo de la investigación con la calidad de los programas académicos en Derecho, dada la enorme exigencia que supone alcanzar las categorías A1 y A, sería más equilibrado agregar también a los programas que tienen grupos de investigación en categoría $\mathrm{B}$, a efectos de formarse un juicio definitivo en la materia. De hecho, Colciencias valora de modo notable el logro de la categoría «B, puesto que para el programa de financiamiento de estudios de doctorado las instituciones elegibles deben disponer, por lo menos, de un grupo de investigación de categoría «B, lo que supone que esa categoría ofrece altas condiciones de calidad para el desarrollo de la investigación ${ }^{18}$. Así mismo, si se examinan los requisitos demandados por Colciencias para obtener la categoría «B, desde luego, puede concluirse que un grupo en ese escalafón posee ya altos niveles de desarrollo de la inves- tigación ${ }^{19}$. En la misma dirección, el cna, al considerar los criterios para la acreditación de alta calidad de los programas académicos de Maestría y Doc- torado, estipula que deben estar respaldados por grupos de investigación en categorías «A1, «A o $\ll \mathrm{B}^{20}$. Por ende, puede concluirse también por los

investigación, desarrollo tecnológico o de innovación y de reconocimiento de investigadores del Sistema Nacional de Ciencia, Tecnología e Innovación 2017», cit., p. 86.

18 Colciencias. "Convocatoria doctorados nacionales 2015", disponible en www. colciencias.gov.co], consultado en enero de 2018, p. 3, apartado 5.4.

19 Colciencias. «Modelo de medición de grupos de investigación, desarrollo tecnológico o de innovación y de reconocimiento de investigadores del Sistema Nacional de Ciencia, Tecnología e innovación 2017», cit., p. 86. 
argumentos referidos, que los grupos de investigación en categoría B exponen parámetros de alta calidad. Ahora, en concordancia con lo explicado, se aplaza para más adelante el análisis de la correlación entre calidad educativa y desarrollo de la investigación en Colombia, de tal manera que se puedan combinar los datos de grupos de investigación en las categorías «A1, «A y «B.

Con grupos de investigación de una clase «A1 o «A comparecen un total de 23 instituciones de educación superior privadas y cuatro universidades públicas. En otras palabras, entre las que tenían esta tipología de grupos, la excelencia en investigación la alcanzaron en un 85\% programas académicos en Derecho de instituciones de educación superior privadas y en un $15 \%$ instituciones de educación superior públicas. Esto sí, ya de manera concluyente, denota un atraso de los programas de Derecho de la universidad pública respecto de la privada en materia investigativa. Desde luego, el panorama podrá examinarse de nuevo cuando se combinen los grupos de excelencia («A1 y «A) con los grupos de alta calidad («B). Empero, hasta ahora sería imperativa la asignación de mayores recursos económicos a la universidad pública con el propósito específico de mejorar la investigación, lo cual supondría una mejor gestión de los recursos de las regalías y la no desviación de grandes cantidades de recursos públicos destinados a programas gubernamentales, que terminan financiando a universidades privadas.

Reanuda el ranking la Universidad Santo Tomás de Bogotá $\left(110^{\circ}\right)$, con cuatro grupos en categoría «A. Aparece entonces la Libre de Cali $\left(12 .^{\circ}\right)$, que además de los tres grupos en «A, posee otros dos de la misma índole compartidos, el primero en asocio con una institución, por lo que se le ha dado un valor de 0,5 , el segundo en alianza con dos instituciones, por lo que se le ha otorgado un valor de 0,33, es decir, puede afirmarse que tiene 3,83 grupos de investigación en categoría «A, mientras que uno de sus tres grupos en $\mathrm{C}$ también es compartido. Con tres grupos de investigación en categoría A se encuentra la Libre de Bogotá $\left(\mathbf{1 3}^{\circ}\right)$. Después arriban tres universidades: Sergio Arboleda Bogotá $\left(14 .^{\circ}\right)$, Sabana $\left(15 .^{\circ}\right.$ ) y Medellín (16..$^{\circ}$ ) con dos grupos en A, que se diferencian por el número de grupos en $\mathrm{B}$ y $\mathrm{C}$ que los acompañan. Enseguida, con un grupo en A: Manizales $\left(17 .^{\circ}\right)$, que tiene otro en B; Cartagena $\left(18 .^{\circ}\right)$, con cuatro grupos en $\mathrm{C}$, tres de ellos compartidos; del Sinú en Bogotá $\left(19 .^{\circ}\right)$ y la Surcolombiana $\left(19 .^{\circ}\right)$, con un grupo en C.

20 Ministerio de Educación Nacional. Consejo Nacional de Acreditación. «Lineamientos para la acreditación de alta calidad de programas de Maestría y Doctorado ", disponible en www.cana.gov.co, Bogotá, mayo de 2010, p. 27, consultado en enero de 2018. 
Ranking de la investigación en los programas de Derecho

Tabla II. Posiciones 11 a 19

\begin{tabular}{|c|c|c|c|c|c|}
\hline Ubicación & Institución & $\mathbf{A}$ & B & $\mathbf{C}$ & $\mathbf{R}$ \\
\hline $11 .^{\circ}$ & Santo Tomás Bogotá & 4 & 0 & 0 & 0 \\
\hline $12 .^{\circ}$ & Libre de Cali & 3.83 & 0 & 2.5 & 0 \\
\hline $13 .^{\circ}$ & Libre de Bogotá & 3 & 1 & 2 & 2 \\
\hline $14 .^{\circ}$ & Sergio Arboleda Bogotá & 2 & 2 & 0 & 0 \\
\hline $15 .^{\circ}$ & Sabana & 2 & 1 & 1 & 0 \\
\hline $16 .^{\circ}$ & Medellín & 2 & 0 & 0 & 0 \\
\hline $17 .^{\circ}$ & Manizales & 1 & 1 & 0 & 0 \\
\hline $18 .^{\circ}$ & Cartagena & 1 & 0 & 2.5 & 0 \\
\hline $19 .^{\circ}$ & Sinú Montería & 1 & 0 & 1 & 0 \\
\hline $19 .^{\circ}$ & Surcolombiana & 1 & 0 & 1 & 0 \\
\hline
\end{tabular}

Fuente: Elaboración propia.

Después emerge la Libre de Barranquilla $\left(21^{\circ}\right)$, que tiene un grupo adicional en $\mathrm{C}$ compartidos con dos instituciones. En el ranking con un grupo insular en A surgen: de la Costa $\left(2 .^{\circ}\right)$; Manuela Beltrán de Bogotá (22. $\left.{ }^{\circ}\right)$; Santo Tomás de Tunja (22. ${ }^{\circ}$ ); Simón Bolívar de Barranquilla (22..$^{\circ}$; Simón Bolívar de Cúcuta $\left(\mathbf{2 2}^{\circ}\right)$. Cierra la lista de grupos en A la Universidad San Buenaventura de Cali $\left(27 .^{\circ}\right)$ con uno, el cual es compartido con otra institución, por lo que es registrado con un 0,5 .

Ranking de la investigación en los programas de Derecho

Tabla III. Posición 21 a 27

\begin{tabular}{clcccc}
\hline Ubicación & Institución & A & B & C & R \\
\hline $21^{\circ}$ & Libre de Barranquilla, & $\mathbf{1}$ & 0 & 0,33 & 0 \\
$22^{\circ}$ & de la Costa & $\mathbf{1}$ & 0 & 0 & 0 \\
$22^{\circ}$ & Manuela Beltrán,Bogotá & $\mathbf{1}$ & 0 & 0 & 0 \\
$22^{\circ}$ & Santo Tomás Tunja & $\mathbf{1}$ & 0 & 0 & 0 \\
$22^{\circ}$ & Símon Bolívar Barranquilla & $\mathbf{1}$ & 0 & 0 & $\mathbf{1}$ \\
$22^{\circ}$ & Simón Bolívar Cúcuta & $\mathbf{1}$ & 0 & 0 & 0 \\
$27^{\circ}$ & San Buenaventura Cali & 0,5 & 0 & 0 & 0 \\
\hline
\end{tabular}

Fuente: Elaboración propia.

En cuanto a la distribución regional de los programas de Derecho con grupos de investigación en categorías A1 y A, ellos asoman en Bogotá (11), Barranquilla (4), Cali (3), Cartagena (1), Cúcuta (1), Manizales (2), 
Medellín (1), Montería (1), Neiva (1), Santa Marta (1) y Tunja (1) ${ }^{21}$. En Colombia, además de Bogotá, capital y ciudad más populosa del país, en su orden, las cinco ciudades más importantes se completan con Medellín, Cali, Barranquilla y Bucaramanga. Bogotá aloja al 41\% de los programas con gru- pos de investigación en categorías A1 y A, lo que habla de la concentración del conocimiento y de la investigación. Mientras que Medellín presenta el 4\%, pese a ser la segunda ciudad en importancia nacional; Barranquilla, con un reporte destacado, mostraba un 15\%; Cali llegaba al 11\%; mientras Bucaramanga, la quinta ciudad, con claro atraso, no presentaba ningún gru- po en el nivel analizado. Por último, un conjunto de capitales de departa- mento menores comparecían con una meritoria representación, entre las que se destacaban Manizales con dos grupos, Cartagena, Cúcuta, Montería, Neiva, Santa Marta y Tunja con uno.

El programa de Derecho de la Universidad Colegio Mayor del Rosario es el único con cinco grupos de investigación en categorías A1 y A, a lo que se agregaba el Externado que posee también cinco grupos en $\mathrm{A}$, aunque ninguno es A1. A su vez, los programas de las universidades Católica de Colombia y Santo Tomás de Bogotá constituyen los únicos con cuatro grupos de investigación en categoría A en el registro de grupos de investigación de Colciencias, con primacía de la Universidad Católica, ya que uno de ellos es A1. Por ende, estas cuatro facultades ejercen un liderazgo muy significativo de la investigación en el ámbito del Derecho en Colombia, con una cantidad bastante elevada de grupos de investigación en las máximas categorías del escalafón de Colciencias. En realidad, disponer de cinco o de cuatro grupos de investigación en categorías A1 y A representa una cota muy alta.

Una cuestión de interés es que la calidad educativa suele ser correlacionada con los altos costos de matrícula. Sobre la relación de la calidad, basada solo en el indicador de los resultados de investigación, la tendencia tradicional que asociaba altos valores de matrícula con la calidad educativa se resquebraja. De los 27 programas de Derecho que muestran resultados de excelencia, 15 corresponden a programas de bajo costo de matrícula, 11 a programas con altos valores de matrícula y dos a programas con costos intermedios. Si se combinaran los datos anteriores con los programas con grupos en categoría B, la tendencia advertida se acentuaría; es decir, aunque los programas con costos altos de matrícula figuran entre los mejores, sería mayoritaria la presencia de programas con bajo costo de matrícula. La anterior es una luz dentro de un sistema

21 Varios programas funcionan en poblaciones cercanas a Medellín; todos ellos fueron ubicados en esa ciudad por pertenecer a su área metropolitana. Lo mismo sucedió con programas de Chía respecto de Bogotá y de Floridablanca con relación a Bucaramanga. 
educativo que ha sido profundamente excluyente, puesto que habría una oferta de buenos programas a costos asequibles para la clase media. Todo esto significaría que la transformación que se ha producido en el país a raíz de la introducción de los sistemas de verificación de la calidad (registro calificado y acreditación), no solo vino a mejorar la calidad de la educación jurídica, por lo menos en lo que al indicador de la investigación respecta, sino que además ha producido resultados en el escenario de una cierta democratización de la educación con calidad.

De otra parte, el análisis que se ha desarrollado en este escrito se basa de manera predominante en datos cuantitativos, como también el sistema de la clasificación de Colciencias tiene una base cuantitativa, aun cuando cada categoría con la que es calificado un grupo de investigación refleja factores o criterios de orden cualitativo, que ilustran la calidad de la investigación producida. Sin embargo, debe señalarse también en términos críticos que resulta claro que varios programas se han concentrado en estructurar un grupo de investigación bastante fuerte, con lo que resultan bien calificados y ubicados en el ranking, pero que no están en condiciones de atender las distintas necesidades en materia de investigación de los diferentes campos del Derecho. De acuerdo con las definiciones tradicionales, son cuatro las grandes áreas del Derecho: privado, penal, público y laboral, las cuales pueden ser divididas en innumerables subáreas, además de la de estudios interdisciplinarios (filosofía, sociología, historia y antropología jurídicas, sobre todo), aun cuando estos últimos pueden aparecer de modo transversal entre las áreas tradicionales. Pero conforme a lo explicado, muchos programas con uno o dos grupos no están en condiciones de cubrir todas las áreas señaladas como principales. En algunos otros casos, la investigación de todas las áreas del Derecho aparece reunida en un solo grupo, pero en ese evento, es evidente que el grupo no funciona como tal, pues las enormes diferencias sobre problemas de investigación, marcos teóricos, etc. lo hacen imposible. Más adelante, al revisar los grupos en categorías $\mathrm{B}$ y $\mathrm{C}$, desde luego sumados a los de nivel A, deberá examinarse cuáles programas tienen un número de grupos de investigación igual o superior a cuatro que, por tanto, de manera más o menos integral tengan una cobertura sistemática y especializada de los grandes campos del Derecho.

A continuación en el ranking comienzan su presencia grupos en categoría B que, conforme a lo explicado, son representativos de niveles de alta calidad. Allí se encuentran con dos grupos en categoría B la Corporación Universitaria Republicana $\left(28 .^{\circ}\right)$ y la Universidad eafit $\left(28{ }^{\circ}\right)$.

Aquí, es muy meritorio que una institución universitaria, como es el caso de la Corporación Universitaria Republicana, logre ubicar dos grupos en esta categoría de alta calidad, pues las instituciones universitarias al no poder ofrecer 
programas de maestría y de doctorado se encuentran en evidente desventaja para llegar a ese resultado, lo cual hace mucho más loable su trabajo de investigación. En realidad, lo que se supone que distingue a una universidad de una institución universitaria, de acuerdo con la excluyente ley de educación superior colombiana, son los niveles alcanzados por la investigación. Empero, lo paradójico es que una institución universitaria, como la Corporación Universitaria Republicana (Bogotá), aparezca con resultados de investigación en una cota bastante por encima de un número muy considerable de universidades y tenga apenas el carácter de institución universitaria. Desde luego, para Colombia se trata de la primera institución universitaria en desarrollo de la investigación en Derecho. El efecto inverso de esa paradoja es que, a su vez, existen muchas universidades con programas de Derecho y resultados bastante pobres en materia de investigación, que no hacen honor al carácter académico que tienen.

Después se encuentran programas de Derecho con un solo grupo de investigación en categoría B, que pertenecen a las siguientes universidades: Gran Colombia de Bogotá $\left(\mathbf{3 0}^{\circ}\right)$, acompañada de cuatro grupos de investigación en categoría C. Por su parte, la Autónoma de Colombia $\left(31 .^{\circ}\right)$ ingresa al escalafón con otros tres grupos en $\mathrm{C}$, aunque dos de ellos son compartidos, por lo que solo son registrados dos grupos más. De manera análoga, concurren las universidades de la Amazonía (31. ${ }^{\circ}$ ), de Antioquia (31. ${ }^{\circ}$ ), Autónoma de Bucaramanga $\left(31 .^{\circ}\right)$ y Libre de Cúcuta $\left(310^{\circ}\right)$ que suman otros dos grupos en $\mathrm{C}$. La Autónoma del Caribe (36. ${ }^{\circ}$ ) presenta dos grupos adicionales en $\mathrm{C}$, pero uno de ellos es compartido con dos instituciones, lo que le agrega 0,33. Con posterioridad, con un grupo adicional en C comparecen las universidades: Autóno- ma Latinoamericana $\left(37 .^{\circ}\right)$, de Nariño $\left(37 .^{\circ}\right)$ y Santo Tomás de Bucaramanga $\left(37 .^{\circ}\right)$. Solo aperadas con el grupo en B están las siguientes instituciones: Bolivariana de Medellín (40. ${ }^{\circ}$ ); Católica de Oriente (40. ${ }^{\circ}$ ); Cooperativa de Pas- to $\left(40 .^{\circ}\right)$; Libre de Pereira (40. ${ }^{\circ}$ ); Militar Nueva Granada (40. $)$; Santiago de Cali $\left(\mathbf{4 0} .^{\circ}\right)$, Ibagué $\left(40 .^{\circ}\right)$ y el Tecnológico Comfenalco de Cartagena $\left(40 .^{\circ}\right)$.

Un total de 47 programas de Derecho reportan resultados de investigación de excelencia y de alta calidad en esa área, al registrar grupos de investigación en categorías A1, A y B. Tales programas, con resultados sobresalientes en cali- dad, representan el 26\% del total de programas en Derecho de Colombia, alrededor de una cuarta parte, habida cuenta que a noviembre de 2017 eran 184 las carreras de Derecho. El 74\% de los programas de Derecho están por fuera de este escenario de calidad. Las instituciones de educación superior privadas son 40, el $85 \%$ de este grupo, mientras las públicas son siete, el $15 \%$, con lo cual la tendencia ya advertida de mayor desarrollo de la investigación en las instituciones privadas no solo es confirmada, sino que en verdad se acentúa. En la distribución regional se encuentra una presencia de programas 
en Bogotá (16), Medellín (6), Cali (4), Barranquilla (5), Bucaramanga (2), Cartagena (2), Manizales (2), Pasto (2), Cúcuta (1), Florencia (1), Ibagué (1), Montería (1), Neiva (1), Pereira (1), Santa Marta (1) y Tunja (1), lo que sigue evidenciado el predominio de Bogotá, pero muestra un desarrollo bastante aceptable de las ciudades que le siguen en importancia y un número total de 16 ciudades con programas destacados.

Ranking de la investigación en los programas de Derecho

Tabla IV. Posiciones 28 a 40

\begin{tabular}{|c|c|c|c|c|c|}
\hline Ubicación & Institución & $\mathbf{A}$ & B & $\mathrm{C}$ & $\mathbf{R}$ \\
\hline $28 .^{\circ}$ & Republicana & 0 & 2 & 0 & 0 \\
\hline $28 .^{\circ}$ & EAFIT & 0 & 2 & 0 & 0 \\
\hline $30 .^{\circ}$ & Gran Colombia Bogotá & 0 & 1 & 4 & 0 \\
\hline $31 .^{\circ}$ & Antioquia & 0 & 1 & 2 & 0 \\
\hline $31 .^{\circ}$ & Autonóma Bucaramanga & 0 & 1 & 2 & 0 \\
\hline $31^{\circ}$ & Autónoma de Colombia & 0 & 1 & 2 & 0 \\
\hline $31 .^{\circ}$ & Amazonía & 0 & 1 & 2 & 0 \\
\hline $31 .^{\circ}$ & Libre de Cúcuta & 0 & 1 & 2 & 0 \\
\hline $36 .^{\circ}$ & Autónoma del Caribe & 0 & 1 & 1,33 & 0 \\
\hline $37 .^{\circ}$ & Nariño & 0 & 1 & 1 & 0 \\
\hline $37 .^{\circ}$ & Santo Tomás Bucaramanga & 0 & 1 & 1 & 0 \\
\hline $37 .^{\circ}$ & Autónoma Latinoamericana & 0 & 1 & 1 & 2 \\
\hline $40 .^{\circ}$ & Bolivariana de Medellín & 0 & 1 & 0 & 2 \\
\hline $40 .^{\circ}$ & Católica de Oriente & 0 & 1 & 0 & 0 \\
\hline $40^{\circ}$ & Cooperativa de Pasto & 0 & 1 & 0 & 0 \\
\hline $40 .^{\circ}$ & Libre de Pereira & 0 & 1 & 0 & 0 \\
\hline $400^{\circ}$ & Militar Nueva Granada & 0 & 1 & 0 & 0 \\
\hline $40 .^{\circ}$ & Santiago de Cali & 0 & 1 & 0 & 0 \\
\hline $40 .^{\circ}$ & Comfenalco Cartagena & 0 & 1 & 0 & 0 \\
\hline $400^{\circ}$ & Universidad de Ibagué & 0 & 1 & 0 & 0 \\
\hline
\end{tabular}

Fuente: Elaboración propia.

Más adelante, apoyados por grupos de investigación en categoría C, los cuales comparecen en cantidades variables, emergen varios programas de Derecho. Entre ellos, con dos grupos en categoría C, la Católica Luis Amigó (48 ${ }^{\circ}$; Pedagógica y Tecnológica de Colombia $\left(\mathbf{4 8}^{\circ}\right)$ y Remington de Medellín $\left(\mathbf{4 8}^{\circ}\right)$. Así mismo, la Libre de Cartagena $\left(\mathbf{4 8}^{\circ}\right)$, que a un grupo en categoría $\mathrm{C}$ agrega dos de esa misma clase en asocio con la Universidad de Cartagena, por lo que puede decirse que tiene dos grupos en C. A su vez, la Universidad del Atlántico $\left(\mathbf{5 2}^{\circ}\right)$, además de un grupo en $\mathrm{C}$ tiene otro compartido con dos instituciones que recibe un valor de 0,33 . 
Las instituciones de educación que reportan un grupo en categoría $\mathrm{C}$ son: Antonio Nariño de Bogotá (53..$^{\circ}$ ); Agraria de Colombia $\left(53 .^{\circ}\right)$; Área Andina de Pereira (53. ${ }^{\circ}$ ); Envigado (53. ${ }^{\circ}$ ); Autónoma de las Américas de Medellín (53. ${ }^{\circ}$; Bolivariana de Bucaramanga $\left(53 .^{\circ}\right)$; del Bosque $\left(53 .^{\circ}\right)$; Boyacá (53..$^{\circ}$; Cauca $\left(53 .^{\circ}\right)$; cesmag $\left(53 .^{\circ}\right)$; Colombo Internacional $\left(53 .^{\circ}\right)$; Cooperativa de Bogotá

$\left(53 .^{\circ}\right)$; Cooperativa de Bucaramanga $\left(53 .^{\circ}\right)$; Cooperativa de Montería (53. ${ }^{\circ}$ ); Cooperativa de Neiva (53..$^{\circ}$; Cooperativa de Santa Marta (53..$^{\circ}$; Francisco de Paula Santander de Cúcuta (53..$^{\circ}$; Francisco de Paula Santander de Ocaña (53. ${ }^{\circ}$ ); Gran Colombia de Armenia (53. ${ }^{\circ}$ ); incca $\left(53 .^{\circ}\right)$; Javeriana de Cali $\left(53 .^{\circ}\right)$, Jorge Tadeo Lozano (54..$^{\circ}$; Juan de Castellanos $\left(54 .^{\circ}\right)$; Libre de Socorro $\left(54 .^{\circ}\right)$; Los Libertadores (53..$\left.^{\circ}\right)$; Magdalena $\left(53{ }^{\circ}\right)$; del Meta $\left(53 .^{\circ}\right)$; Popular del Cesar $\left(53 .^{\circ}\right)$; Rafael Núñez de Barranquilla (53..$^{\circ}$; Rafael Núñez de Cartagena (53. ${ }^{\circ}$ ); Corporación Universitaria de Sabaneta (53. ${ }^{\circ}$ ); San Buenaventura de Medellín (53. ${ }^{\circ}$ ); Santo Tomás de Villavicencio (53..$^{\circ}$; S Sinú de Cartagena (53. $\left.{ }^{\circ}\right)$; Tecnológica del Chocó Diego Luis Córdoba $\left(53 .^{\circ}\right)$; Empresarial Alexander von Humboldt $\left(53 .^{\circ}\right)$ y uniciencia $\left(53 .^{\circ}\right)$. Con un grupo en $\mathrm{C}$ compartido, que reciben un valor de 0,5, la Santo Tomás de Medellín (94. ${ }^{\circ}$ y la Tecnológica Antonio Arévalo en Cartagena -tecnar- $\left(94 .^{\circ}\right)$.

\section{Ranking de la investigación en los programas de Derecho}

Tabla V. Posiciones 48-94

\begin{tabular}{|c|c|c|c|c|c|}
\hline Ubicación & Institución & $\mathbf{A}$ & B & $\mathbf{C}$ & $\mathbf{R}$ \\
\hline $48 .^{\circ}$ & Católica Luis Amigó & 0 & 0 & 2 & 0 \\
\hline $48 .^{\circ}$ & Libre de Cartagena & 0 & 0 & 2 & 0 \\
\hline $48 .^{\circ}$ & Pedagógica y Tecnológica & 0 & 0 & 2 & 0 \\
\hline $48 .^{\circ}$ & Remington Medellín & 0 & 0 & 2 & 0 \\
\hline $52 .^{\circ}$ & Atlántico & 0 & 0 & 1.33 & 0 \\
\hline $53 .^{\circ}$ & Antonio Nariño Bogotá & 0 & 0 & 1 & 0 \\
\hline $53 .^{\circ}$ & Agraria de Colombia & 0 & 0 & 1 & 0 \\
\hline $53 .^{\circ}$ & Área Andina Pereira & 0 & 0 & 1 & 0 \\
\hline $53 .^{\circ}$ & Envigado & 0 & 0 & 1 & 0 \\
\hline $53 .^{\circ}$ & Autónoma de las Américas & 0 & 0 & 1 & 0 \\
\hline $53 .^{\circ}$ & Bosque & 0 & 0 & 1 & 0 \\
\hline $53 .^{\circ}$ & Boyacá & 0 & 0 & 1 & 0 \\
\hline $53 .^{\circ}$ & Cauca & 0 & 0 & 1 & 0 \\
\hline $53 .^{\circ}$ & Bolivariana Bucaramanga & 0 & 0 & 1 & 0 \\
\hline $53 .^{\circ}$ & CESMAG & 0 & 0 & 1 & 0 \\
\hline $53 .^{\circ}$ & Cooperativa Bogotá & 0 & 0 & 1 & 0 \\
\hline $53 .^{\circ}$ & Cooperativa Bucaramanga & 0 & 0 & 1 & 0 \\
\hline $53 .^{\circ}$ & Cooperativa Montería & 0 & 0 & 1 & 0 \\
\hline $53 .^{\circ}$ & Cooperativa Neiva & 0 & 0 & 1 & 0 \\
\hline $53 .^{\circ}$ & Cooperativa Santa Marta & 0 & 0 & 1 & 0 \\
\hline
\end{tabular}




\begin{tabular}{|c|c|c|c|c|c|}
\hline $53 .^{\circ}$ & Francisco de Paula Santander Cúcuta & 0 & 0 & 1 & \\
\hline $53 .^{\circ}$ & Javeriana de Cali & 0 & 0 & 1 & \\
\hline $53 .^{\circ}$ & Sabaneta & 0 & 0 & 1 & \\
\hline $53 .^{\circ}$ & Colombo Internacional & 0 & 0 & 1 & \\
\hline $53 .^{\circ}$ & Empresarial A. Humboldt & 0 & 0 & 1 & \\
\hline $533^{\circ}$ & Francisco de Paula Santander Ocaña & 0 & 0 & 1 & \\
\hline $53 .^{\circ}$ & Gran Colombia Armenia & 0 & 0 & 1 & \\
\hline $53 .^{\circ}$ & INCCA & 0 & 0 & 1 & \\
\hline $53 .^{\circ}$ & Jorge Tadeo Lozano & 0 & 0 & 1 & \\
\hline $53 .^{\circ}$ & Juan de Castellanos & 0 & 0 & 1 & \\
\hline $53 .^{\circ}$ & Libre de Socorro & 0 & 0 & 1 & \\
\hline $53 .^{\circ}$ & Los Libertadores & 0 & 0 & 1 & \\
\hline $53 .^{\circ}$ & Magdalena & 0 & 0 & 1 & \\
\hline $53 .^{\circ}$ & Del Meta & 0 & 0 & 1 & \\
\hline $53 .^{\circ}$ & Popular del Cesar & 0 & 0 & 1 & \\
\hline $53 .^{\circ}$ & Rafael Núñez Barranquilla & 0 & 0 & 1 & \\
\hline $53 .^{\circ}$ & Rafael Núñez Cartagena & 0 & 0 & 1 & \\
\hline $53 .^{\circ}$ & Tecnológica Diego Luis Córdoba & 0 & 0 & 1 & \\
\hline $53 .^{\circ}$ & San Buenaventura Medellín & 0 & 0 & 0,5 & \\
\hline $53 .^{\circ}$ & Santo Tomás Villavicencio & & & & \\
\hline $53 .^{\circ}$ & Sinú Cartagena & & & & \\
\hline $89 .^{\circ}$ & Santo Tomás Medellín & & & & \\
\hline $9 .^{\circ}$ & TECNAR Cartagena Fuente: elabora & & & & \\
\hline
\end{tabular}

Fuente: Elaboración propia.

Para el reconocimiento como grupo categoría $\mathrm{C}$, Colciencias exige unos requisitos mínimos: 1. Un indicador de grupo mayor que cero; 2. Un producto tipo Top o A; 3. Un producto de apropiación social del conocimiento; 4. Un producto tipo A o B de actividades de formación de conocimiento; 5. Dos años de existencia ${ }^{22}$.

En total son identificados 90 programas de Derecho que disponen del respaldo de grupos de investigación categorizados en A, B y/o C. Esto representa el 49\% del total de programas académicos en Derecho del país, puesto que puede recordarse que en total eran 184 activos a noviembre de 2017. A su vez, una gran cantidad de facultades y programas de Derecho no poseen absolutamente ningún grupo de investigación con categoría registrado en Colciencias. Sería un global de 94 programas de Derecho que no alcanzarían a tener ningún grupo de

22 Colciencias. «Modelo de medición de grupos de investigación, desarrollo tecnológico o de innovación y de reconocimiento de investigadores del Sistema Nacional de Ciencia, Tecnología e Innovación 2017», cit., p. 86. 
investigación en el área del conocimiento jurídico con escalafón. Esto, en Colombia, representa el $51 \%$ del total de carreras o programas de grado que se imparten en Derecho, más de la mitad, lo que representa un balance muy negativo.

Se había mencionado páginas atrás que era prudente examinar qué programas de Derecho tenían al menos cuatro grupos de investigación de respaldo o apoyo, en categorías A1, A, B o C, puesto que al menos existen en los estudios de Derecho cuatro grandes áreas del conocimiento: público (constitucional y administrativo), penal, privado (civil y comercial) y laboral. Puede darse por descontado que, en términos cualitativos, no debería bastar que un programa se concentre en sumar uno o dos grupos de investigación con una alta categoría en el escalafón. La conclusión anterior, pues aunque tales grupos serían muy fuertes, como se explicó en detalle atrás, en todo caso el programa tendría áreas fundamentales del Derecho descuidadas, o una concentración de todos sus investigadores en un grupo para obtener una categoría alta sin que esa unidad funcione, por la diversidad de materias involucradas, como un auténtico grupo (Briceño, Ricardo, 2018) .

Así las cosas, con cuatro grupos de investigación o más en las cuatro categorías definidas por Colciencias, esto es, A1, A, B o C, concurren las siguientes instituciones de educación superior, respecto de las cuales se indica su posición en el ranking general: Universidad Colegio Mayor de Nuestra Señora del Rosario $\left(\mathbf{1}^{\circ}\right)$, Universidad de los Andes (2. $\left.{ }^{\circ}\right)$, Universidad Católica de Colombia (3. ${ }^{\circ}$, Universidad Nacional de Colombia $\left(4^{\circ}\right)$, Universidad Javeriana de Bogotá $\left(5^{\circ}\right)$, Universidad Externado de Colombia $\left(100^{\circ}\right)$, Universidad Santo Tomás de Bogotá $\left(11 .^{\circ}\right)$, Universidad Libre de Cali $\left(12 .^{\circ}\right)$, Universidad Libre de Bogotá $\left(13 .^{\circ}\right)$, Universidad Sergio Arboleda en Bogotá $\left(14 .^{\circ}\right)$, Universidad La Sabana $\left(15 .^{\circ}\right)$ y Universidad La Gran Colombia de Bogotá $\left(\mathbf{3 0} .^{\circ}\right)$.

De conformidad con la variable antes explicada, un total de 12 programas académicos tendrían niveles sobresalientes de desarrollo de la investigación con una amplia cobertura de las áreas del Derecho involucradas. Son los programas que han procurado un desarrollo más o menos equilibrado y, sobre todo, integral de la investigación. Esto representa un exiguo 6,5\% del total de programas académicos en Derecho. Este dato adicional, que introduce una mirada diferente y, claro está, crítica, sobre la manera como se organizan los grupos de investigación de Derecho, expondría resultados muy deficientes para el progreso de la investigación jurídica y sociojurídica en Colombia.

A la par, como puede verse con facilidad, varios programas, entre los más importantes y destacados en el ranking, pues son cuatro clasificados entre los diez primeros, tienen un número muy pequeño de grupos de investigación y desaparecen del listado anterior. 
Si se construyera un modelo ideal de investigación en Derecho, de acuerdo con las pautas metodológicas de Max Weber mencionadas en la introducción de este trabajo, se tendría lo siguiente: un modelo básico de investigación en un programa académico de Derecho, con al menos cinco años de funcionamiento, debería disponer como mínimo de cuatro grupos de investigación en categorías A1, A, B o C, con un número plural significativo de profesores vinculados. De tal manera, se cubrirían todas las áreas principales del Derecho. No son contemplados los grupos de investigación apenas reconocidos, dado que son aún muy incipientes. También se piensa que debe haber un número importante de docentes investigadores por grupo, puesto que un grupo puede alcanzar incluso la categoría más alta con una cantidad muy baja de docentes investigadores (dos o tres profesores), pero ello estaría muy lejos de implicar que la cultura de la investigación ha permeado a todo el programa y que el estudio e innovación del conocimiento es común al cuerpo docente en general.

Ahora, al comparar los resultados del ranking con el modelo anterior, que no es exigente en demasía, el balance general de la investigación jurídica y sociojurídica en Colombia arrojaría un saldo bastantenegativo.

Varias instituciones de educación superior presentan tan solo grupos de investigación que apenas fueron reconocidos, pero no categorizados. Entre ellos los programas pertenecientes a las siguientes instituciones: del Área Andina de Valledupar $\left(91^{\circ}\right)$; Universitaria de Popayán $\left(91 .^{\circ}\right)$; Tecnológica deBolívar $\left(91 .^{\circ}\right)$; Navarra (91. $\left.{ }^{\circ}\right)$; U. de Colombia $\left(91 .^{\circ}\right)$.

Los grupos de investigación que cuentan apenas con reconocimiento, en verdad, son representativos de un estado muy incipiente de desarrollo. En resumen, los requisitos principales están referidos a 1. Antigüedad de un año; 2. La participación de dos investigadores; 3 . El registro de solamente un producto de nuevo conocimiento de cualquiera clase.

\section{Ranking de la investigación en los programas de Derecho}

Tabla VI. Posición 91

\begin{tabular}{clllll}
\hline Ubicación & Institución & A & B & C & R \\
\hline $91 .^{\circ}$ & Área Andina de Valledupar & 0 & 0 & 0 & $\mathbf{1}$ \\
$91 .^{\circ}$ & Tecnológica de Bolívar & 0 & 0 & 0 & $\mathbf{1}$ \\
$91 .^{\circ}$ & de Popayán & 0 & 0 & 0 & $\mathbf{1}$ \\
$91 .^{\circ}$ & Navarra & 0 & 0 & 0 & $\mathbf{1}$ \\
$91 .^{\circ}$ & U. de Colombia & 0 & 0 & 0 & $\mathbf{1}$ \\
\hline
\end{tabular}

Fuente: Elaboración propia. 
De conformidad con lo anterior, un total de 95 programas de Derecho presentan resultados de investigación, con grupos que tienen una categoría en el escalafón o que fueron apenas reconocidos. Una cifra bastante elevada, que alcanza al $48 \%$ de los programas en Derecho, no posee resultados como para siquiera lograr integrar un grupo de investigación, con un único producto de investigación de cualquier clase. Desde luego, podría haber instituciones que hayan optado por no utilizar el sistema de Colciencias, lo cual es absolutamente legítimo, por cuanto no existe obligación legal de registrar los grupos en la plataforma de Colciencias o participar en sus evaluaciones, pues la obligación legal consiste en demostrar que la investigación es una actividad del programa, lo que puede hacerse al margen de Colciencias. Sin embargo, ese tipo de situaciones, si existen, son excepcionales. También puede haber programas muy nuevos, que no han contado con el tiempo indispensable para desarrollar la investigación docente, lo que es probable, demandaría de dos o tres años. Igualmente, puede haber programas con algún grupo destacado, pero que ha sido registrado como perteneciente a un área del conocimiento más genérica, como por ejemplo humanidades, a fin de usarlo para soportar varios programas académicos a la vez. Empero, aún considerando las hipótesis anteriores, es evidente que la cantidad de programas activos de Derecho que no presentan resultados ni siquiera mínimos en investigación como para al menos llegar a obtener el reconocimiento de un grupo de investigación es muyalta.

Aunque debe reconocerse que el éxito logrado por el sistema de aseguramien- to de la calidad (registro calificado y acreditación), de manera evidente, ha repercutido en el desarrollo destacado de varias instituciones en materia de investigación jurídica y sociojurídica, también los resultados anteriores ponen en duda la eficacia del registro calificado. Esto es, además, contradictorio. El registro calificado parece que funcionó respecto de muchos programas, pero a la par la ausencia de resultados en investigación con relación a otros progra- mas pone en duda la coherencia de las acciones de control de la calidad.

Algunas instituciones universitarias, pese a no contar con el carácter académico de universidades, tuvieron resultados muy destacados en materia de investigación, todo ello pese a que, como regla general, no tienen competencia legal para impartir programas de maestría y doctorado, de los cuales se derivan varios productos que entregan puntajes considerables de acuerdo con los criterios deColciencias. Entonces, aquellasinstituciones universitariasquealcanzaron a ubicar grupos de investigación en categorías A1, A y B obtuvieron resultados muy meritorios, los cuales contrastan con los obtenidos por muchas instituciones de educación superior con el carácter de universidades. Esto demuestra, de nuevo, que los avances en investigación son una consecuencia de 1. Voluntad y compromiso institucional; 2. Políticas de investigación idóneas; 3. Dirección y gestión adecuada dela investigación; 4. Existencia de una cultura 
de investigación; 5. Políticas y acciones eficaces para la divulgación de los resultados; 6 . Investigadores formados, con experiencia y comprometidos; y 7. Recursos financieros de apoyo.

Siendo que, pese a la concurrencia de varios factores adversos, hay algunas instituciones universitarias con muy buenos resultados en investigación, es claro que la distinción entre universidades e instituciones universitarias presente en la ley colombiana es injustificada ${ }^{23}$. Se supone que el rasgo fundamental que diferencia a los dos tipos de instituciones es la investigación, dado que en las universidades debería de alcanzar cotas mayores. Empero, como ha sido indicado, comparecen algunas instituciones universitarias con muy buenos resultados en investigación para Derecho, de excelencia o de alta calidad $\mathrm{y}$, en contraste, hay varias universidades que exponen resultados muy débiles.

Otra cuestión adicional, que deriva en una contradicción notable con las pautas establecidas en la ley, también incongruente con las exigencias de los procesos de registro calificado y de acreditación en alta calidad que demandan de resultados en investigación, es la distinción conceptual promovida por algunos sectores entre universidades de investigación y universidades de docencia. Los datos del ranking en Derecho demuestran que una división como la anterior es artificial. Ella daría lugar a pensar, lo que sería un absurdo mayor y una contradicción patente con la discutible división entre universidades e instituciones universitarias, que hay instituciones universitarias de investigación e instituciones universitarias dedocencia.

En definitiva, un ranking de institucionesuniversitarias, incluyendosolo a aquellas con resultados de excelencia y de alta calidad, estaría conformado por:

\section{INSTITUCIÓN:}

1. Corporación Universitaria Republicana

2. Tecnológico confenalco Cartagena

$\begin{array}{cc}\text { A1 } & \text { B } \\ 0 & 2 \\ 0 & 1\end{array}$

Las anteriores instituciones universitarias están presentes en Bogotá (1) y Cartagena (1). Las demás instituciones universitarias que muestran resultados investigativos solo tienen grupos en categoría C.

Cabe interrogarse si los resultados precedentes son exclusivos de Derecho o si, al contrario, son comunes a otros programas de tales instituciones. En el primer caso, se trataría de un éxito puntual y aislado de sus programas de

23 Ley 30 de 28 de diciembre de 1992, Diario Oficial, n. ${ }^{\circ}$ 40.700, de 29 de diciembre de 1992, disponible en https://www.cna.gov.co/1741/articles-186370_ley_3092.pdf. 
Derecho; en el segundo, sería evidente que son producto de los factores institucionales que se han mencionado párrafos atrás como generadores de éxito en materia de investigación, por tanto, más o menos comunes y genera- les a todos sus programas ${ }^{24}$.

Pues bien, en el caso de la Corporación Universitaria Republicana, ella cuenta con cinco grupos en B y uno en C; en lo que concierne a la Fundación Universitaria Tecnológico confenalco Cartagena, ella posee seis grupos de investigación, uno en categoría A y cinco en categoría B. En consecuencia, la respuesta sería que no son resultados aislados, ya que la mayoría de las instituciones analizadas disponen de resultados excelentes respecto de la mayoría de sus grupos. De allí, que sea válido afirmar que la investigación depende más de la concurrencia de un conjunto de factores institucionales que pueden hacerla altamente exitosa.

De otra parte, resulta interesante correlacionar los datos del ranking en investigación con el registro de programas de Doctorado. Las universidades Colegio del Rosario $\left(1 .^{\circ}\right)$, de Los Andes $\left(2 .^{\circ}\right)$, Nacional de Colombia (4..$\left.^{\circ}\right)$, Javeriana $\left(5 .^{\circ}\right)$, Externado de Colombia $\left(10{ }^{\circ}\right)$, Santo Tomás de Bogotá $\left(11 .^{\circ}\right)$, Libre de Bogotá $\left(13 .^{\circ}\right)$ y Sergio Arboleda de Bogotá $\left(14 .^{\circ}\right)$ poseen programas de Doctorado en Derecho, lo cual resulta del todo coherente con su ubicación dentro del ranking, puesto que se trata de facultades de Derecho que exponen resultados de excelencia en investigación jurídica y sociojurídica, de acuerdo con los parámetros de Colciencias. En cambio, es incongruente que los programas de las universidades Católica de Colombia $\left(3 .^{\circ}\right)$, Libre de Cali $\left(12 .^{\circ}\right)$ y Sabana $\left(15 .^{\circ}\right)$ no ofrezcan Doctorado en Derecho, dado que tienen un número plural de grupos de investigación de excelencia y alta calidad. Es también paradójico el caso de la Universidad Libre, donde la seccional más fuerte en investigación, la de Cali, no posee Doctorado, más cuando en esa ciudad no se brinda ningún programa de ese tipo, mientras que la sede de Bogotá sí lo ofrece. Desde luego, lo que resulta relevante en términos de calidad es la primera observación precedente, esto es, que las facultades de Derecho que tienen niveles de excelencia en investigación hospedan doctorados en Derecho.

Después, en todo caso con resultados de excelencia en investigación, pues tienen al menos un grupo en categoría A, aunque sin tanta fortaleza como las diez primeras universidades, ofrecen formación doctoral las universidades de Medellín (16. ${ }^{\circ}$, que tiene un doctorado en Derecho Procesal Contemporáneo

24 1. Voluntad y compromiso institucional; 2. Políticas de investigación idóneas; 3. Dirección y gestión adecuada de la investigación; 4. Existencia de una cultura de investiga- ción; 5. Políticas y acciones eficaces para la divulgación de los resultados; 6. Investigadores formados, con experiencia y comprometidos; y 7. Recursos financieros de apoyo. 
y otro genérico en Derecho; del Norte $\left(6 .^{\circ}\right)$ y, así mismo, la Santo Tomás de Tunja (22..$^{\circ}$ que oferta un programa especializado en Derecho Público. De los anteriores programas, los doctorados en Derecho, es decir, genéricos, de las universidades de Medellín y del Norte tienen menos de cuatro grupos de investigación clasificados para apoyarlos, pese a que los programas abarcarían todas las áreas del Derecho. Los programas de doctorado en procesal y público de las universidades de Medellín y Santo Tomás de Tunja, en cambio, al ser especializados son congruentes con un desarrollo también especializado en investigación. El programa de doctorado de la Facultad de Derecho de la Universidad de Antioquia (31. ${ }^{\circ}$ ) aparece rezagado en el ranking, pues no relaciona grupos de investigación en A, pero registra un grupo en categoría B, que significa un nivel de alta calidad y, así mismo, tiene dos grupos de investigación categorizados en $\mathrm{C}$, pero no alcanza a tener cuatro grupos de investigación con escalafón.

En cuanto a las exigencias del cna para el reconocimiento de alta calidad de los programas de Doctorado, en lo que se refiere al requisito de las categorías de los grupos de investigación, debe recordarse que el documento del cna para programas de posgrado demanda la existencia de grupos, esto es en plural, con categorías A1, A o B, es decir, dos o más grupos como los indicados. Por tanto, en lo referido únicamente a este componente puede decirse que los pro- gramas de Doctorado de las universidades Colegio del Rosario (1. $\left.{ }^{\circ}\right)$, Los An- des (2. $)$, Nacional de Colombia $\left(4 .^{\circ}\right)$, Javeriana de Bogotá $\left(5^{\circ}\right)$, Externado de Colombia $\left(10 .^{\circ}\right)$, Santo Tomás de Bogotá $\left(11 .^{\circ}\right)$, Libre de Bogotá $\left(13 .^{\circ}\right)$, Sergio Arboleda de Bogotá $\left(14 .^{\circ}\right)$ y Medellín (16. $\left.{ }^{\circ}\right)$ cumplen con creces las condicio- nes requeridas para una acreditación de alta calidad (Restrepo, Guillermo, 2018).

En otro escenario, resulta de interés ponderar los resultados del ranking de investigación en Derecho, relacionándolos con la acreditación de alta calidad de los programas académicos de pregrado en Derecho. En ese sentido, el cna, en lo que concierne a la acreditación de alta calidad para los programas de pregrado en Derecho, desdeluego, debe considerar la investigación, por cuanto se trata de uno de los factores que integran la noción de alta calidad. No es el único factor que decide la acreditación de alta calidad, pero es uno de ellos.

En total, existen 40 programas de Derecho con acreditación de alta calidad ${ }^{25}$. Estos programas representan el 22\% del total de programas académicos de

$25 \mathrm{Al}$ respecto, Ministerio de Educación Nacional. snies, cit. El programa de derecho de la Universidad Javeriana en Cali figura dos veces, pero solo se ha contabilizado una vez. El programa de la Universidad Colegio Mayor del Rosario figura como Jurisprudencia pero, como es obvio, se tuvo en cuenta como Derecho. 
Derecho existentes en el país, dado que debe recordarse que eran 184 a noviembre de 2017. A la par, esto significa que el 78\% de los programas de Derecho notienen acreditación, pero cumplen con las condiciones de calidad básicas o esenciales, según el Ministerio de Educación Nacional, puesto que este les otorgó registro calificado. Los programas acreditados, en el mismo orden en el que aparecen en el ranking de investigación de Derecho, con un número plural de grupos de excelencia o de alta calidad de acuerdo a los parámetros de Colciencias y del cna que se han relacionado, es decir, con grupos en A y/ o B, son:

Colegio Mayor de Nuestra Señora del Rosario (1. $\left.{ }^{\circ}\right)$, Universidad de los Andes $\left(2 .^{\circ}\right)$, Universidad Católica de Colombia $\left(3 .^{\circ}\right)$, Universidad Nacional de Colombia $\left(4 .^{\circ}\right)$, Universidad Javeriana de Bogotá $\left(5 .^{\circ}\right)$, Universidad Externado de Colombia $\left(\mathbf{1 0}^{\circ}\right)$, Universidad Santo Tomás de Bogotá $\left(11 .^{\circ}\right)$, Universidad Libre de Cali (12..$^{\circ}$, Universidad Libre de Bogotá $\left(13 .^{\circ}\right)$, Universidad Sergio Arboleda de Bogotá $\left(14 .^{\circ}\right)$, Universidad de la Sabana $\left(15 .^{\circ}\right)$, Universidad de Medellín (16. ${ }^{\circ}$ ), Universidad de Manizales $\left(17 .^{\circ}\right)$ y Universidad eafit $\left(\mathbf{2 8} .^{\circ}\right)$.

Los 14 programas anteriores, que muestran frutos muy destacados en materia de investigación, puesto que disponen de dos o más grupos en categorías A1, A y/o B, a la par gozan de acreditación de alta calidad, lo que evidencia una coherencia elevada entre los niveles de investigación y los resultados de la acreditación, lo cual ilustra la labor del cna. Y, en este punto, debe recordarse lo que Colciencias y el propio cna han indicado sobre el significado de poseer grupos en categoría A1, A y/o B en materia de calidad. Así mismo, aunquela acreditación se otorga por el cumplimiento sobresaliente de varios factores, entre los cuales la investigación es apenas uno de ellos, no cabe duda que siendo uno tan importante, la congruencia comentada es muy satisfactoria. Nótese, además, que de los diez primeros programas de Derecho con resultados óptimos en materia de investigación se encuentran acreditados apenas seis.

Concurren varios programas acreditados que, sin embargo, no tienen un número plural de grupos en categorías A1, A y/o B, por lo que no fueron relacionados, habida cuenta de que en el documento de acreditación de posgrados el cna habla de grupos en esas categorías, lo que supone varios. El criterio anterior, que el cna valida para posgrados, podría ser aún más pertinente para pregrados, puesto que los primeros suelen ser programas con cierto nivel de especialidad, mientras que los segundos son generales.

$\mathrm{Al}$ margen de la lista de programas de Derecho acreditados por el Ministerio de Educación Nacional, casi que no hay ningún programa de Derecho que merecería, al menos en virtud a su producción investigativa, estar acreditado, 
esto es, por contar con un número plural de grupos en categorías A1, A y/o B. El único que podría ser mencionado es el programa de Derecho de la Corporación Universitaria Republicana (28. ${ }^{\circ}$ ), que precisamente es el único programa no acreditado que tiene un número plural de grupos de investigación en los niveles superiores, con dos grupos en categoría B.

En cambio, los resultados de la acreditación no guardan una relación de coherencia clara con las evidencias sobre investigación, respecto de un pequeño conjunto de programas que, si bien están acreditados, no poseían resultados de excelencia o de alta calidad de acuerdo con los parámetros de Colciencias y del mismo cna. Es decir, estos programas carecían del todo de grupos de investigación en categorías A1, A o B. No es la misma situación que recién se planteó, en la que se comentaba de programas acreditados que no tenían un número plural de grupos en A1, A o B; la observación es que no poseen ningún grupo en categorías de excelencia o de alta calidad. Por ende, tales programas aparecían, en su mayoría, apoyados por un único grupo en categoría C.

El Banco Mundial había planteado que el modelo de expansión de la educación superior recomendable, distinto al seguido por Colombia de modo predominante, no debía ser el de la creación de nuevas instituciones de educación superior con programas del todo nuevos, sino que debía fundarse en el desarrollo de las instituciones y los programas ya existentes, los cuales debían extenderse a otras localidades y regiones del país a fin de aprovechar su trayectoria ya consolidada ${ }^{26}$. Sin embargo, la revisión de los datos sobre investigación en Derecho no parece darle la razón. Conforme a estos, salvo contadas excepciones como, por ejemplo, los casos de la Universidad Libre o de la Universidad Sergio Arboleda, hubo muchos programas de Derecho que en sus domicilios principales alcanzaron resultados positivos; en cambio, en otros pertenecientes a seccionales o extensiones, obtuvieron resultados muy deficientes. Lo anterior sin contar programas de Derecho de baja calidad en investigación, con resultados malos o pésimos para sus sedes principales, que exponían balances igual o más negativos en otras seccionales o extensiones. En contraste, algunas instituciones nuevas lograron con sus programas, desde luego también nuevos, resultados bastante positivos (Motta, Ricardo, 2018) .

Es claro que los grupos de investigación localizados en una ciudad, que en las solicitudes de registro calificado con bastante frecuencia son presentados como apoyo de programas de grado o pregrado situados en otras regiones del país,

26 Banco Mundial. La enseñanza superior. Las lecciones derivadas de la experiencia, Washington, 1995, disponible en http://documentos.bancomundial.org/curated/es/ 274211468321262162/La-ensenanza-superior-las-lecciones-derivadas-de-la-experiencia, pp. 27 y ss. 
en realidad no están en condiciones de brindar «a larga distancia» el apoyo necesario para el desarrollo adecuado de los mismos.

Así mismo, pese a los muy buenos resultados en investigación que ilustran la actividad de muchos programas de Derecho e, igualmente, pese a que es evidente que el sistema de aseguramiento de la calidad del Estado ha tenido un éxito inusitado al impulsar la investigación en un número muy considerable de programas de Derecho donde ella simplemente era del todo inexistente, también es claro, al menos en lo que al rubro de investigación respecta, que las observaciones formuladas sobre las deficiencias y desigualdades en la calidad de la educación superior colombiana se mantienen vigentes ${ }^{27}$.

\section{CONCLUSIONES}

De las observaciones y datos que han sido expuestos, se derivan varias conclusiones a tener en cuenta.

1. Existen varios programas de Derecho con resultados extraordinarios en materia de investigación, los cuales lideran en el país los desarrollos y avances de la ciencia jurídica.

2. Es relativamente bajo el número de programas de Derecho que muestran resultados de excelencia o de alta calidad, esto es, de grupos en categorías A1, A y B.

3. Es bastante marcado el desarrollo superior de la investigación de excelencia y de alta calidad en instituciones de educación superior privadas.

4. Concurre un atraso importante de la investigación en los programas de Derecho de las universidades públicas, pues salvo contados y sobresalientes casos, el balance general comprueba que no tienen presencia entre los programas con resultados de excelencia y de alta calidad. Esto demanda de recursos públicos del Estado para apoyar el avance sustancial de la investigación en varias universidades públicas.

5. En términos generales, la investigación en los programas de Derecho es deficiente, puesto que no solo la tasa de programas que no tienen grupos de excelencia y de alta calidad es elevada, sino que, además, es

27 Germán Silva García. El mundo real de los abogados y de la justicia, t. i, «La profesión jurídica», Bogotá, Instituto Latinoamericano de Servicios Legales Alternativos -ilsa- y Externado, 2001, pp. 39 y ss. 
mayoritario el porcentaje de programas de Derecho que no tienen grupos de investigación en ninguna de las categorías de Colciencias.

6. Existe una concentración relativa de los programas de Derecho con resultados de excelencia y alta calidad en Bogotá, que no es tampoco muy marcada.

7. Es muy escaso el número de programas que presentan cuatro o más grupos de investigación, para permitir una cobertura integral de las grandes áreas del Derecho.

8. La tendencia tradicional que apuntaba una correlación entre altos cos- tos de matrícula y calidad de la educación, al menos en razón al indica- dor de la investigación, se ha quebrado de modo significativo, con el efecto de democratizar el acceso a la educación con calidad y, así mis- mo, reducir las tendencias a la exclusión social de los más vulnerables en términos socioeconómicos.

9. La distinción entre universidades de investigación y universidades de docencia, según los resultados vistos en la investigación en Derecho, parece del todo artificial y carente de sentido.

10. Los programas de varias instituciones universitarias tienen desarrollos muy importantes en investigación, pese a que no son universidades, lo que desdice de las diferencias legales entre las dos clases de instituciones. Esto es corroborado con el hecho de que estas instituciones en otras áreas del conocimiento tienen también muy buenos resultados.

11. En cambio, muchas universidades son bastante débiles en investiga- ción, pese a que tienen ese carácter.

12. En términos generales, existe coherencia entre los desarrollos investigativos en el ámbito de los programas de Derecho y la creación y desarrollo de programas de doctorado en Derecho.

13. En término generales, la congruencia entre acreditación de alta calidad e investigación es elevada, pese a algunas excepciones por lo que al factor de investigación corresponde, pues algunos pocos programas con resultados bastante deficientes han alcanzado la acreditación.

14. Habría un número bastante considerable de programas en Derecho que tienen registro calificado, pero que no tienen grupos de investigación en ninguna de las categorías de Colciencias, con las implicaciones que ello puede contraer en materia de calidad. 
15. El modelo propuesto por el Banco Mundial de crecimiento de la oferta en educación superior a partir de la extensión de las instituciones y programas que estaban en funcionamiento antes de la reforma educativa de 1992, al menos respecto de la mayoría de las instituciones que lo utilizaron, parece haber fracasado, por lo que atañe a los programas de Derecho y conforme a los resultados en investigación.

16. La renovación del registro calificado de programas académicos debía comprender exigencias más elevadas en los resultados de investigación, al igual que en el caso de los programas que pierden el registro calificado y vuelven a presentarse como nuevos, puesto que la comparación con un modelo ideal de investigación básico es muy desfavorable para la realidad de los programas de Derecho.

17. Aun cuando ha habido mejoras sustanciales e importantes, examinados los programas de Derecho en el renglón de la investigación, es confirmada la tesis sobre la existencia de grandes desigualdades y deficiencias en la calidad de la educación superior.

\section{REFERENCIAS}

Banco Mundial.La enseñanza superior. Las lecciones derivadas de la experiencia, Washington, 1995, disponible en http://documentos.bancomundial.org/curated/es/2742114 68321262162/La-ensenanza-superior-las-lecciones-derivadas-de-la-experiencia.

Bocanegra Acosta, Henry. «La enseñanza del derecho y la formación de los abogados», en Revista Republicana, $\mathrm{n} .{ }^{0}$ 12, enero-junio de 2012, disponible en http:// ojs.urepublicana.edu.co/index.php/revistarepublicana/article/view/5o/46.

Botero Bernal, Andrés. «Un marco de comprensión del avance de la investigación jurídica en Colombia», en Academia. Revista sobre la Enseñanza del Derecho, año 5, n. $^{\circ}$ 10, 2007, disponible en http://www.derecho.uba.ar/publicaciones/rev_acade$\mathrm{mia} /$ revistas/10/un-marco-de-comprension-del-avance-de-la-investigacionjuridica-en-colombia.pdf.

Briceño, Ricardo y Otros (2018). Enfoque diferencia en la gestion social: Investigación colaborativas con comunidades Negras, indigenas y personas con discapacidad. ISBN: 978-958-48-5628-9. Fondo de publicaciones Corporación Universitaria Republicana

Ceballos Bedoya, María Adelaida. «Educación jurídica, abogados y movilidad social en Colombia», Universidad Nacional de Colombia, 2016, disponible en http:// www.bdigital.unal.edu.co/54799/. 
Curvelo, Jose y Otros. (2018). Las cuentas invisibles del trabajo de las mujeres. ISBN: 978-958-48-5629-6. Fondo de publicaciones Corporación Universitaria Republicana.

Colciencias. Listado resultados finales -781 de 2017-, Grupos (Para Consulta), disponible en [www.colciencias.gov.co], revisado en enero de 2018.

Colciencias. "Convocatoria doctorados nacionales 2015», disponible en www. colciencias.gov.co, consultado en enero de 2018.

Colciencias. «Modelo de medición de grupos de investigación, desarrollo tecnológico o de innovación y de reconocimiento de investigadores del Sistema Nacional de Ciencia, Tecnología e Innovación 2017», disponible en www.colciencias.gov.co, consultado en enero de 2018.

Cossio Acevedo, Nora Alba. «Informe final de la investigación: "Pertinencia e impacto social de la investigación jurídica: el caso del programa de derecho de la Universidad de Antioquia 1992-2009" ", en Estudios de Derecho, vol. 70, n. ${ }^{0} 156,2013$, disponible en https://aprendeenlinea.udea.edu.co/revistas/index.php/red/article/ view/20037.

Instituto Colombiano para el Fomento de la Educación Superior -icfes-. Educación superior. Compendio de normas, Bogotá, icfes, 1995.

Larrauri Torroela, Ramón. «La educación jurídica como campo de investigación desde una conceptualización epistemológica», en Universitas. Revista de Filosofia, Derecho y Politica,.$^{\circ}$ 3, 2005/2006, disponible en http://universitas.idhbc.es/no3/ 03-05_larrauri.pdf.

Ley 30 de 28 de diciembre de 1992, Diario Oficial, n. ${ }^{\circ}$ 40.700, de 29 de diciembre de 1992,disponibleenhttps://www.cna.gov.co/1741/articles-186370_ley_3092.pdf.

Motta, Ricardo (2018). El Biocentrismo en el derecho al agua en practicas ecológicas en la Sabana de Bogotá. ISBN: 978-958-48-5625-8. Fondo de publicaciones Corporación Universitaria Republicana.

Restrepo, Guillermo (2018). Fundamentos Constitucionales del Sistema Penal Colombiano. ISBN: 978-958-48-5626-5. Fondo de publicaciones Corporación Universitaria Republicana.

República de Colombia. Ministerio de Educación Nacional. Consejo Nacional de Acreditación. "Lineamientos para la acreditación de alta calidad de programas de Maestríay Doctorado», disponibleen www.cana.gov.co, Bogotá, mayo de 2010.

Silva García, Germán. El mundo real de los abogados y de la justicia, t. i, «La profesión jurídica», Bogotá, Instituto Latinoamericano de Servicios Legales Alternativos ilsa- y Externado, 2001. 
Silva García, Germán. El mundo real de los abogados y de la justicia, t. iv, «Las ideologías profesionales», en Bogotá, Instituto Latinoamericano de Servicios Legales Alternativos -ilsa- y Externado, 2001.

Silva García, Germán. «Prospectivas sobre la educación jurídica», en Rogelio Pérez Perdomo y Julia Cristina Rodríguez (coords.). La formación jurídica en América Latina, Bogotá, Externado,2006.

Torregrosa Jiménez, Norhys Esther y Rodolfo Torregrosa Jiménez. «La investigación sociojurídica una función prioritaria en la formación de los abogados y abogadas del siglo xxi en Colombia», Verba Iuris, n. ${ }^{0}$ 28, julio-diciembre de 2012, disponible en http://www.unilibre.edu.co/verbaiuris/28/Editorial.pdf.

Weber, Max. Economía y sociedad, 2. ${ }^{\text {a }}$ ed., México D. F., Fondo de Cultura Económica, 1992. 